This is a self-archived - parallel published version of this article in the publication archive of the University of Vaasa. It might differ from the original.

\title{
Implementation of an intelligent caravan monitoring system using a simple serial communication protocol for microcontrollers (SSCPM)
}

Author(s): Glocker, Tobias; Mantere, Timo

Title: Implementation of an intelligent caravan monitoring system using a simple serial communication protocol for microcontrollers (SSCPM)

Year: $\quad 2019$

Version: Accepted manuscript

Copyright (C)2019 IEEE. Personal use of this material is permitted. Permission from IEEE must be obtained for all other uses, in any current or future media, including reprinting/republishing this material for advertising or promotional purposes, creating new collective works, for resale or redistribution to servers or lists, or reuse of any copyrighted component of this work in other works.

\section{Please cite the original version:}

Glocker, T., \& Mantere, T., (2019). Implementation of an intelligent caravan monitoring system using a simple serial communication protocol for microcontrollers (SSCPM). In: 2019 1oth International Conference on Information and Communication Systems (ICICS), Irbid, Jordan (pp. 250-255). Institute of Electrical and Electronics Engineers (IEEE). https://doi.og/10.1109/IACS.2019.8809149 


\section{Implementation of an Intelligent Caravan Monitoring System Using a Simple Serial Communication Protocol for Microcontrollers (SSCPM)}

\author{
Tobias Glocker \\ University of Vaasa \\ Email: tglo@uva.fi
}

\author{
Timo Mantere \\ University of Vaasa \\ Email: timan@uva.fi
}

\begin{abstract}
Safety applications play an essential role in our daily life. Without them many accidents would have happened. Especially nowadays, where the amount of traffic increases year by year, safety applications have become an important factor for the vehicle industry. Statistics have shown that the amount of accidents could be remarkably reduced for vehicles with safety systems. However, most of the safety systems are implemented on vehicles but not on trailers.

This paper describes the implementation of an Intelligent Trailer Monitoring System which uses a Simple Serial Communication Protocol (SSCPM) for the communication between the vehicle's board computer and the trailer's monitoring system.
\end{abstract}

\section{INTRODUCTION}

In recent years, there were several accidents with caravans. According to [1] $80 \%$ of the accidents resulted in total destruction of the caravan. The loss of control was the reason for $30 \%$ of the insurance claims. One reason, for the loss of control, is overspeeding. It is recommended, not to drive more than $96.5 \mathrm{kph}$ when drawing a caravan, otherwise a slight force might tip the caravan from one way to another.

A further reason, for the loss of control is overloading, or when the load of the caravan is not balanced. A too high coupling load, could lead to a situation, where the front wheels of the car are loosing the road grip, making the car uncontrollable. Furthermore, a too high load at the back of the caravan, could lead to a situation, where the back wheels of the car are loosing the road grip, making the car uncontrollable, too. It is also important, that the weight of the right side is equal or close to the weight on the left side, otherwise the caravan could tilt over, while driving through a dangerous bend. The age of the tires plays also an essential role for the road safety. Tires, older than five years, should be replaced with new ones, even if they do not show any kind of damage or if the profile depth is still good. Old tires can explode or can get flat, causing that the caravan might skid.

Another hazard that could cause a road accident with caravans, are broken light bulbs. In newspapers, it can be read quite often, that a vehicle with a trailer turned left, but due to a broken indicator bulb, another vehicle was just overtaking it at that moment and crashed into it.
In addition, the carried gas bottles could also cause an explosion if they, or if a gas pipe, becomes leaky for a longer time. This could lead to a serious road accident during the ride.

In this paper, an Intelligent Caravan Monitoring System has been implemented in order to reduce the amount of accidents caused by the previous mentioned reasons.

\section{FUnCTIONALITY OF THE PROPOSED INTELLIGENT CARAVAN MONITORING SYSTEM}

The idea of the Intelligent Caravan Monitoring System is to monitor all kind of electrical devices on board, getting information about the weight, weight distribution, lighting system, temperature, status of the door and the status of windows from the caravan. This system requires, that the car as well as the caravan must be equipped with a board computer that contains a touchscreen. It is to mention, that the board computer of the car acts as a Master while the board computer of the caravan is acting as a Slave. In order to read a certain information (temperature values, lighting issues etc.) or to set certain values, the board computer of the car will sent a certain packet type to the caravan's board computer which will then process the packet. The board computer of the caravan is connected with all kind of electrical devices that can be monitored and controlled by the car's board computer. Cars, that have a trailer hitch, are normally equipped with a 13 pole trailer socket but only eleven poles are used. Fig. 1 presents the trailer socket. Table 1 shows the pin assignment of a 13 pole trailer socket. Two poles of the 13 pole trailer socket are used, for the communication between the car's and caravan's board computer [2]. One wire (pole 12) is used for transmitting the packet, the other wire is used for the common ground (pole 13). When transmitting a packet, each byte of a packet is sent separately. SSCPM [3] has been used to transmit a packet byteby-byte from the car's board computer to the caravan's board computer and vice versa. Fig. 2 illustrates the transmission of the byte value 129 (0x81). Each byte transmission starts with a 100us high level signal followed by a 500us low level signal 
before the eight bits of the corresponding byte are transmitted. It takes around $4.6 \mathrm{~ms}$ to transmit one byte.

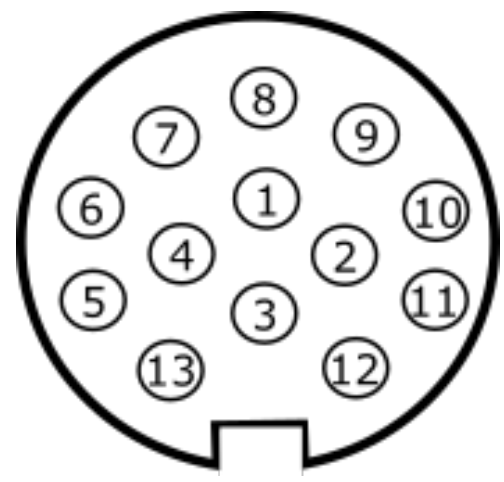

Fig. 1. Trailer Socket.

TABLE I

Pin Assignment of A 13 POLE Trailer Socket

\begin{tabular}{|l|l|}
\hline Pole Number & Consumer \\
\hline 1 & Indicator (left) \\
\hline 2 & Rear Fog Light \\
\hline 3 & GND of Poles 1 to 8 \\
\hline 4 & Indicator (right) \\
\hline 5 & Back Light (right) \\
\hline 6 & Brake Light \\
\hline 7 & Back Light (left) \\
\hline 8 & Backup Lamp \\
\hline 9 & Positive Pole \\
\hline 10 & Positive Pole for Charging \\
\hline 11 & not assigned (GND of Pole 10) \\
\hline 12 & not assigned \\
\hline 13 & GND of Poles 9 to 12 \\
\hline
\end{tabular}

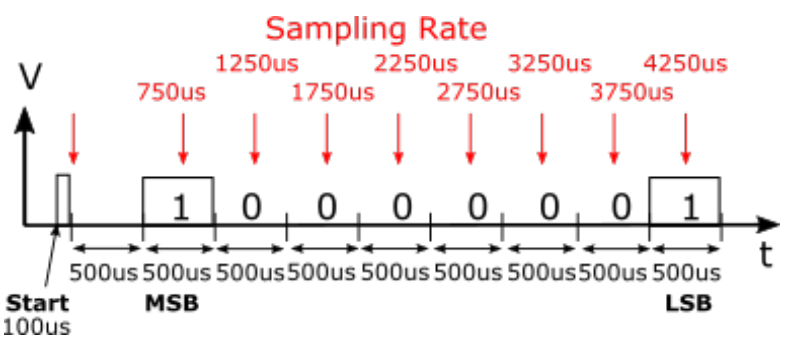

Fig. 2. SSCPM Signal for the transmission of one byte (value 0x81).

In the proposed system there are nine different packet types. Control Packets with and without value, Authentication Packet, Safety Packet, Reply Packets, Trailer Info Packet, Trailer Weight Packet, Trailer Supply Packet and Driver Assistant Packet. The Control Packets and the Authentication Packet are sent from the car's board computer to the caravan's board computer. Based on the request of the received Control Packet, the caravan's board computer replies either with a Safety Packet, Reply Packets, Trailer Info Packet, Trailer Weight Packet or Driver Assistant Packet. Fig. 3 shows the format of the packets. A Control Packet is used to request a Safety Packet in certain time intervals or when an indicator is set.

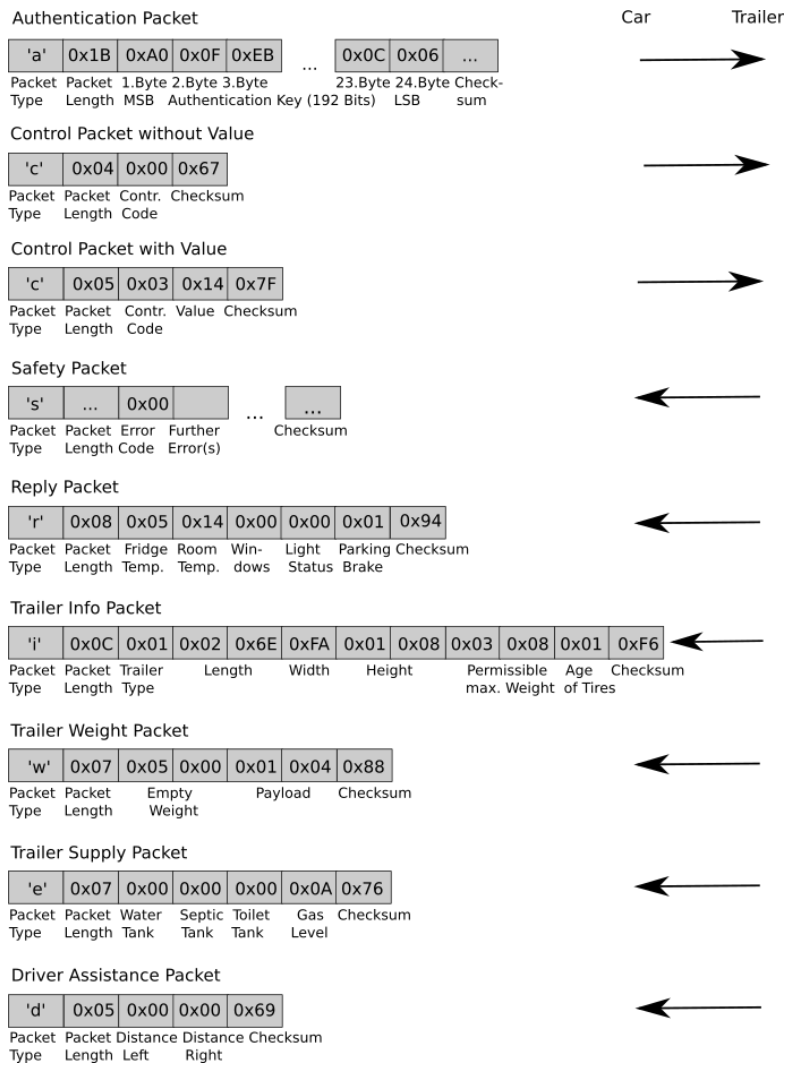

Fig. 3. Packet Formats.

In case of lighting and tire problems, such as an open door or open window(s), the Safety Packet contains the error code(s) which will be sent to the car's board computer in order to inform the driver about the problem. It displays the safety warning message on the screen and generates an acoustic warning signal. Through the Control Packets the driver can also send control commands to the caravan's board computer. Typical control commands are the adjustment of the fridge temperature, air conditioning, turning on/off the light in the caravan as well as opening and closing electronic windows. The Reply Packet contains all the control values of the caravan and it will be sent from the caravan's to the car's board computer after a request from a Control Packet. There is also a Trailer Info Packet, that contains information about the dimensions of the trailer, the maximum permissible weight and the age of the tires. In addition, there is also a Trailer Weight Packet that includes the empty trailer weight and the trailer payload. The Trailer Supply Packet delivers the water tank level, septic tank level, toilet tank level and the amount of gas. Furthermore, the Driver Assistance Packet transfers the values received from the parking sensors. It is to mention, that the Reply Packet, Trailer Info Packet, Trailer Weight Packet and the Driver Assistance Packet are also requested by the Control Packet. As soon as the driver puts the car into reverse, the board computer of the car will request continuously a Driver Assistance Packet from the caravan's board computer.

The Authentication Packet contains a 192 bit authentication 
TABLE II

Control Codes For the CONTROL PACKeT

\begin{tabular}{|l|l|}
\hline Description & Control Code \\
\hline Request Safety Packet & 0x00 \\
\hline Request Reply Packet & 0x01 \\
\hline Request Trailer Info Packet & 0x02 \\
\hline Request Trailer Weight Packet & 0x03 \\
\hline Request Trailer Supply Packet & 0x04 \\
\hline Request Driver Assistance Packet & 0x05 \\
\hline Adjust fridge temperature & 0x06 \\
\hline Adjust temperature of air conditioning & 0x07 \\
\hline Radiators (on/off) & 0x08 \\
\hline Window Control (left side) & 0x09 \\
\hline Window Control (right side) & 0x0A \\
\hline Window Control (front side) & 0x0B \\
\hline Window Control (rear side) & 0x0C \\
\hline Window Control (roof) & 0x0D \\
\hline Light Control & 0x0E \\
\hline Tighten/release electrical brake & 0x0F \\
\hline Release Reversing Lock for the brake & 0x10 \\
\hline Fresh Water Tank Level & 0x11 \\
\hline Septic Tank Level & 0x12 \\
\hline Toilet Tank Level & 0x13 \\
\hline Gas Bottle Monitoring & 0x14 \\
\hline
\end{tabular}

TABLE III

ERror Codes For the SAFETy PACKET

\begin{tabular}{|c|c|}
\hline Description & Error Code \\
\hline No fault & $0 \times 00$ \\
\hline Indicator (left) & $0 \mathrm{x} 01$ \\
\hline Indicator (right) & $0 \times 02$ \\
\hline Brake light (left) & $0 \times 03$ \\
\hline Brake light (right) & $0 \times 04$ \\
\hline Back light (left) & $0 \times 05$ \\
\hline Back light (right) & $0 \times 06$ \\
\hline Rear Fog light (left) & $0 \times 07$ \\
\hline Rear Fog light (right) & $0 \times 08$ \\
\hline Backup Lamp (left) & $0 \times 09$ \\
\hline Backup Lamp (right) & $0 \mathrm{x} 0 \mathrm{~A}$ \\
\hline Electrical parking brake (tighten) & 0x0B \\
\hline Reversing Lock (released) & $0 \mathrm{x} 0 \mathrm{C}$ \\
\hline Tire pressure (left) & 0x0D \\
\hline Tire pressure (right) & $0 \mathrm{x} 0 \mathrm{E}$ \\
\hline Tire pressure (2nd tire left) & $0 \mathrm{x} 0 \mathrm{~F}$ \\
\hline Tire pressure (2nd tire right) & $0 \times 10$ \\
\hline Window opened (left side) & $0 \times 11$ \\
\hline Window opened (right side) & $0 \times 12$ \\
\hline Window opened (front side) & $0 \times 13$ \\
\hline Window opened (rear side) & $0 \times 14$ \\
\hline Window opened (roof) & $0 \times 15$ \\
\hline Door opened & $0 \times 16$ \\
\hline Gas Sensor Alarm & $0 \times 17$ \\
\hline More Weight on Corner (Left Front) & $0 \times 18$ \\
\hline More Weight on Corner (Right Front) & $0 \times 19$ \\
\hline More Weight on Corner (Left Rear) & $0 \times 1 \mathrm{~A}$ \\
\hline More Weight on Corner (Right Rear) & $0 \mathrm{x} 1 \mathrm{~B}$ \\
\hline More Weight on Front & $0 \times 1 C$ \\
\hline More Weight on Back & $0 \times 1 \mathrm{D}$ \\
\hline More Weight on Left Side & $0 \times 1 E$ \\
\hline More Weight on Right Side & $0 \mathrm{x} 1 \mathrm{~F}$ \\
\hline Overweight of Trailer Coupling Load & $0 \times 20$ \\
\hline Problem with the Brakes & $0 \times 21$ \\
\hline
\end{tabular}

code which is sent to the trailer's board computer. Only when the transmitted authentication code matches with the stored authentication key in the caravan's board computer, then a
Trailer Info Packet will be sent to the car's board computer. Furthermore, the caravan's board computer is then ready for processing the received Control Packets. If the car's board computer will not receive a Trailer Info Packet after sending the authentication message, then the authentication failed. In Table 2, the control codes from the Control Packet are listed. Table 3 contains the error codes.

The authentication process is needed to protect a parked caravan from data manipulation or thieves. Without an authentication process, almost every person could connect the vehicle with a parked caravan and could change the temperature settings, turning off the fridge or even opening the windows through the car's board computer. To ensure that this will not happen, the board computer of the caravan generates a 192 bit random number, the so called authentication number, and displays it. This number must be edited and saved in the car's board computer, to establish the communication with the board computer of the caravan. The authentication number is valid until a new authentication number is generated on the caravan's board computer. In case, the caravan's board computer receives three wrong authentication numbers in a row, it will not process any authentication packet until the warning message displayed on the screen is confirmed with the caravan's board computer password. It is important to emphasize, that different packets are used because some information, the caravan dimensions for example, is only needed once.

To this system belongs also a Weight Monitoring System that warns the driver, if the load in the caravan is not balanced. This system is described in the next section.

\section{Weight Distribution System}

In order to achieve a safe driveability, the load of the caravan must be distributed in a certain way.

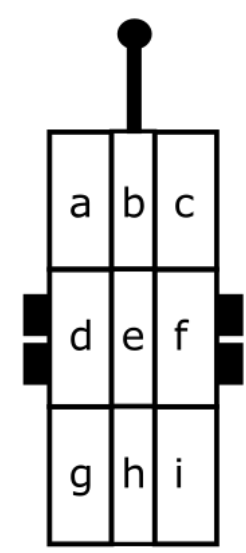

Fig. 4. Trailer Weight Distribution.

According to [4], experiments have shown, that a caravan has the highest driving stability, when the coupling load is close to the maximum and when the weight at the left side is close to the weight at the right side. For that reason, a Weight Distribution System (WDS) has been developed. The bottom 
of the caravan is split into nine parts as shown in Fig. 4. It is assumed, that each of the nine parts are equipped with sensors that can determine the weight. In order to determine if the load is equally distributed, the following algorithm has been developed. It is also to mention, that the load of the caravan cannot be exactly equally distributed because not everywhere are storage compartments available or they are already full or too small for a certain load.

The idea of the WDS is to build a 3x3 Weight Distribution Matrix (WDM) from the caravan bottom that is split into nine parts.

$$
W D M=\left[\begin{array}{lll}
a & b & c \\
d & e & f \\
g & h & i
\end{array}\right]
$$

The following Pseudocode describes how the Weight Distribution Algorithm works.

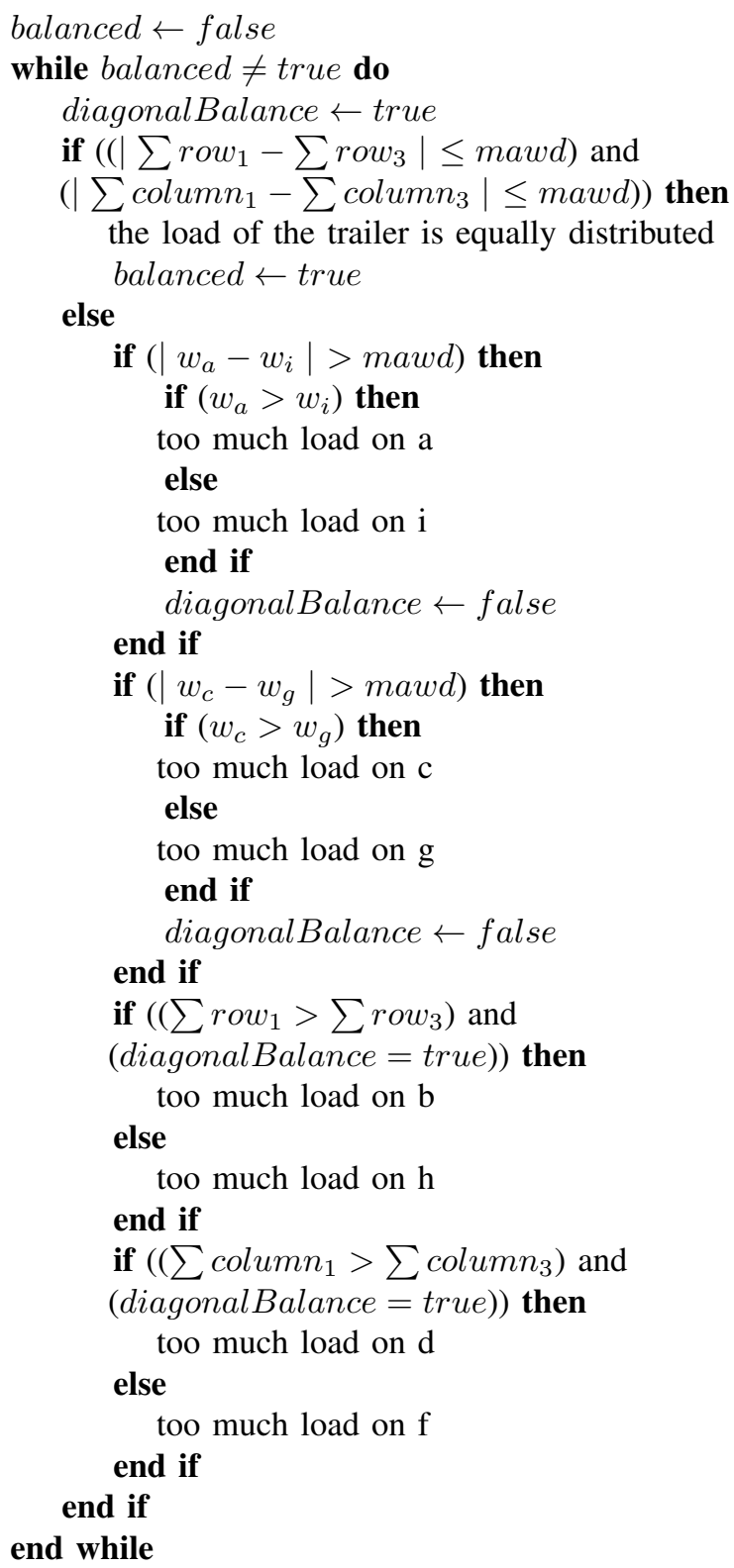

The variable mawd stands for the maximum allowed weight deviation and $w_{a}, w_{i}, w_{c}$ and $w_{g}$ are the variables for the load in the area a, i, c and $\mathrm{g}$.

First, the system will check if the load on the front and back side is equal or in the range of the maximum allowed weight deviation. The same procedure will be done for the left and right side. In case the weight is not equally distributed, the diagonal weight distribution is determined and if it is not equally distributed it will display a warning message on the car's and caravan's board computer. If the diagonal weight distribution is okay, but the weight distribution between the front and back side as well as the weight distribution between the left and right side is not okay, further warning messages are sent and displayed.

\section{IMPLEMENTATION OF THE SYSTEM}

The proposed Intelligent Caravan Monitoring System has been tested on an own implemented system (see Fig. 5). Java has been used for the implementation of the car's board computer and the caravan simulator. Both applications, the car's board computer and the caravan simulator have been running on the same Personal Computer (PC). The SSCPM has been implemented on two MSP-EXP430G2 LaunchPad Development Boards in order to realize the one wire communication between both applications. For the testing, both LaunchPads have been connected with the PC via Universal Serial Bus (USB). Two wires have been used to connect the LaunchPads with each other. One wire is used for the communication and the other wire is used for the common ground. As already mentioned, the Car's Board Computer Application acts as a Master, while the Caravan Simulator Application acts as a Slave. To get information from the trailer, the Car's Board Computer Application will send a Control Packet with the control code to the Caravan Simulator Application, which replies with the corresponding packet.

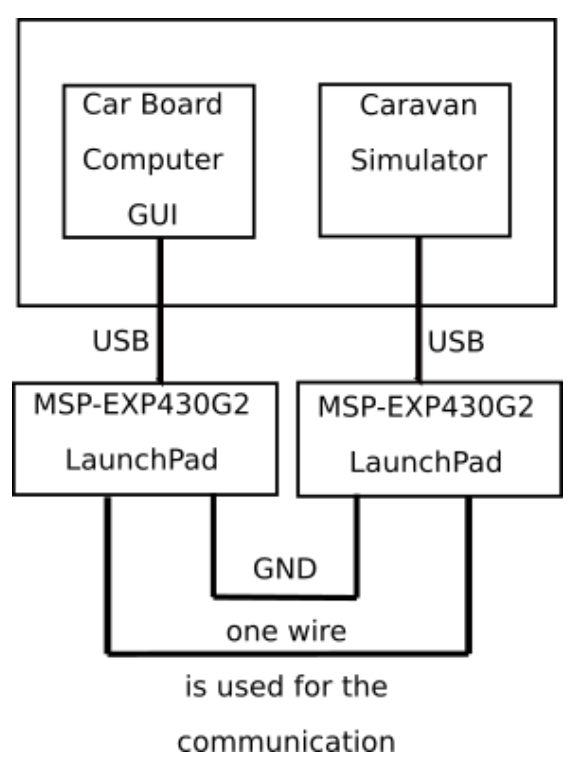

Fig. 5. Overview of the Implemented System. 


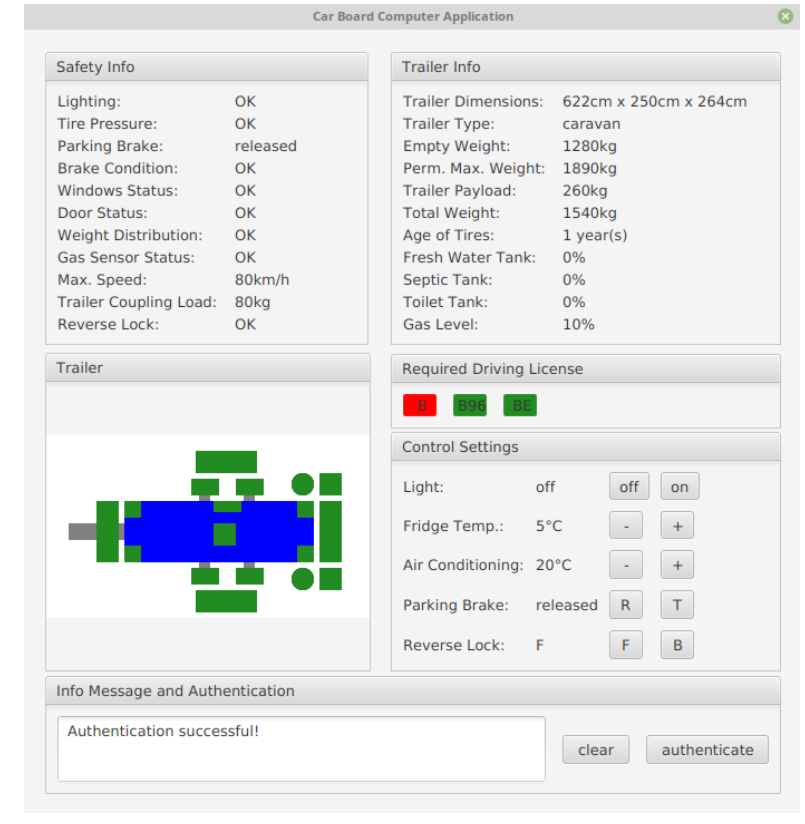

Fig. 6. The Car Board Computer GUI Application.

Fig. 6 shows the Graphical User Interface (GUI) developed with JavaFX. The GUI displays all the trailer information mentioned in section II. The Caravan Simulator Application is currently a console application which will be further developed into a GUI application. Both applications get the information about the car's or caravan's dimensions, empty weight, maximum permissible weight etc. from text files. The GUI will also inform the driver about the distribution of the load (see section III).

\section{FeAtures of The PROPOSED INTELligent CARAVAN MONITORING SYSTEM}

The implemented system has several features that are relevant for safety driving. In addition to the the safety features introduced in section II and the weight distribution introduced in section III, this section describes the functionality of how the system determines the required driving license, the maximum allowed driving speed with the trailer, as well as the amount of gas in a gas bottle.

All member countries of the European Union issue the European Driving License. For cars, there are three different driving licenses available B, B96 and BE [5]. All of them (B, $\mathrm{B} 96$ and $\mathrm{BE}$ ) allow the owner to drive a car up to $3500 \mathrm{~kg}$ but the driving licenses B and B96 limit the total permissible weight of the car and the trailer. Owner's of driving license B can drive a car with a trailer of more than $750 \mathrm{~kg}$ as long as the total permissible weight of the car and trailer does not exceed $3500 \mathrm{~kg}$, otherwise they can only tow a trailer with a maximum permissible weight of $750 \mathrm{~kg}$. Owner's of driving license B96 are allowed to drive a car with a trailer whose permissible weight is more than $750 \mathrm{~kg}$, if the total permissible weight of $4250 \mathrm{~kg}$ is not exceeded. The driving license BE allows to tow trailers up to $3500 \mathrm{~kg}$. The developed system determines the required driving license based on the maximum permissible weight of the car, the maximum permissible weight of the trailer and the total weight of the trailer. First, the maximum permissible weight of the trailer is checked. If the maximum permissible weight of the trailer is equal or below $750 \mathrm{~kg}$, then the car with the trailer can be driven with all driving licenses (B, B96 and BE). Otherwise, the maximum permissible weight of the car and the maximum permissible weight of the trailer are added together. Based on the result, the system will ascertain, if the car with the trailer can be driven with the driving license BE only, B96 and BE only or with all three driving licenses (B, B96 and BE). The system considers also the total weight of the trailer in its decision because due to an overloaded trailer, the next higher driving license could be required.

The maximum allowed driving speed for cars with trailers is $80 \mathrm{~km} / \mathrm{h}$. However, a driving speed of $100 \mathrm{~km} / \mathrm{h}$ is allowed when certain conditions are fulfilled[6].

- the total permissible weight of the car must not be more than $3500 \mathrm{~kg}$

- the car must have an Anti-lock Braking System (ABS)

- the trailer must be suitable for a speed of $100 \mathrm{~km} / \mathrm{h}$

- the tires must have the speed index "L" (max.speed $120 \mathrm{~km} / \mathrm{h}$ )

- the tires must not be older than six years

- total permissible weight of the trailer $\leq \mathrm{y} \times$ empty weight of the car

It is assumed that the factor $\mathrm{y}$ is provided by the trailer manufacturer. The factor depends on the shock absorbers and the brakes of the trailer. Usually the factor has a value between 0.3 and 1.2. This factor must be entered in the car's board computer.

While driving, the driver can also check via the car's board computer, the water tank, septic tank, toilet tank and the amount of gas.

The amount of the gas in the bottle can be determined according the following formula, where $t$ is the total weight of the gas bottle, e is the empty weight of the gas bottle and $x$ is the maximum filling weight of propane [7].

$$
\text { amountOfGas }=\frac{t-e}{x}
$$

\section{CONCLUSION}

In this paper, we have introduced the implementation of an Intelligent Caravan Monitoring System using a Simple Serial Communication Protocol. The main idea of this research, was to build an Intelligent Caravan Monitoring System that informs the driver about the weight, weight distribution, state of the trailer's lighting system, tire pressure and other features. This system gives the driver the opportunity to access and control certain electronic devices such as fridge, air conditioning and so on. The simulators for the car's and caravan's board computer were implemented with Java, while the SSCPM was implemented in C Language. The vehicle's board computer communicates with the caravan's board computer over the SSCPM, that uses two of the free wires of a 13 pole trailer 
socket. One wire is used for the common ground and the other wire is used for the signal transmission. Furthermore, it is to emphasize, that the Controller Area Network Bus (CANBus) has not been used because the developed system should be easily integrated into the current car and caravan systems without major changes.

\section{REFERENCES}

[1] Retreatcaravans.com.au, "Caravan Road Safety: The Top Causes of Caravan Accidents." Internet: www.retreatcaravans.com.au/road-safety-tips/, Oct. 20, 2016 [Oct. 12, 2017].

[2] D. Wächter, "HIGHWAY'S WEBECKE."

Internet: http://www.creativcut.de/stecker.php, 2012-2017 [Oct. 20, 2017].

[3] C. Patil, "Development of a Simple Serial Communication Protocol for Microcontrollers (SSCPM)." International Journal of Scientific and Research Publications, vol. 1, no. 1, Dec. 2011.
[4] Caravaning.de, "PRAXIS-TIPP STÜTZLAST BEIM CARAVANGESPANN. Alles über Stützlast."

Internet: https://www.caravaning.de/zubehoertest/ratgeber- stuetzlastalles-ueber-stuetzlast/, 2013 [Feb. 02, 2019].

[5] TUEV-SUED.de, "Führerschein-Klassen." Internet: https://www.tuev-sued.de/fuehrerschein_pruefung/ fuehrerscheinklassen/, 2019 [Feb. 02, 2019].

[6] TUEV-NORD.de, "100 km/h-Zulassung für Kfz-AnhängerKombinationen." Internet: https://www.tuev-nord.de/de/ privatkunden/verkehr/auto-motorrad-caravan/gespanne-100kmhzulassung/, [Feb. 02, 2019].

[7] Tippscout.de, "Füllstand von Gasflasche ermitteln geht auch ganz genau." Internet: https://www.tippscout.de/fuellstand-gasflascheherausfinden.html, 2017 [Feb. 02, 2019]. 